\title{
Composition and diversity of larval fish in the Indian Ocean using morphological and molecular methods
}

\author{
M. I. G. Rathnasuriya ${ }^{1,2} \cdot$ A. Mateos-Rivera ${ }^{3} \cdot$ R. Skern-Mauritzen ${ }^{3}$ - H. B. U. Wimalasiri ${ }^{2}$ - R. P. P. K. Jayasinghe ${ }^{2}$. \\ J. O. $\mathrm{Krakstad}^{3}$. P. Dalpadado ${ }^{3}$
}

Received: 29 January 2020 / Revised: 16 December 2020 / Accepted: 20 December 2020 / Published online: 1 April 2021

(C) The Author(s) 2021

\begin{abstract}
Although vital in fisheries management, no comprehensive studies on ichthyoplankton have been conducted in Sri Lankan waters in the north central Indian Ocean hitherto. Hence, this study is the first detailed account of diversity and species composition of larval fish based on samples collected during the southwest monsoon in 2018. In total, 80 species belonging to 69 families were identified using morphological and molecular methods targeting the COI gene. The larval fish diversity varied significantly between regions: east (North East, Central East, and South East) and west (North West, South West, and South). In their larval stages, mesopelagic families were associated with the offshore waters whereas demersal and pelagic families were related with shelf regions in the South, South East, and Central East. The larvae of pelagic families are likely dispersed by the South Monsoon Current from the west to the east regions, while demersal fish seem to be confined to the same area as conspecific adults. The most abundant larval species observed were Selar crumenophthalmus, Cubiceps pauciradiatus, and Dipterygonotus balteatus. High abundances of several commercially important larval tuna species were found in the South East, Central East, and South regions indicating that these waters could be important nursery grounds. Furthermore, Callionymus simplicicornis was recorded for the first time in the Indian Ocean, and seven additional species were found new to Sri Lankan waters. The results from this study also highlight the importance of using combined morphological and molecular methods and the need for strengthening fish nucleotide databases in poorly studied areas of the Indian Ocean.
\end{abstract}

Keywords Ichthyoplankton $\cdot$ Species assemblages $\cdot$ COI gene $\cdot$ Spawning $\cdot$ Sri Lankan waters

\section{Introduction}

Ichthyoplankton studies not only provide valuable information on the species richness and spawning activity of fishes but also identify nursery areas for larval fish (Ayala et al. 2016; Ahern et al. 2018). Traditionally, ichthyoplankton samples have been identified based on their meristic, morphometric, and pigmentary characteristics (Rodriguez et al. 2017).

Communicated by S. Ohtsuka

P. Dalpadado

padmini.dalpadado@hi.no

1 Faculty of Fisheries and Ocean Sciences, Ocean University of Sri Lanka, Mahawela Road, Tangalle 82200, Sri Lanka

2 National Aquatic Resources Research and Development Agency (NARA), Crow Island, Colombo 15, 00150, Sri Lanka

3 Institute of Marine Research (IMR), PO Box 1870, Nordnes, N-5817 Bergen, Norway
However, in some cases, the use of available descriptive information to identify ichthyoplankton to species level has led to misidentification (Fox et al. 2005). The recent applications of molecular techniques have to a large degree solved this issue and significantly improved knowledge on ichthyoplankton diversity around the globe. In fact, in the recent years, higher larval diversity has been reported in several studies using molecular methods in temperate and tropical marine ecosystems. In the oligotrophic areas of Sargasso Sea, 154 species from 50 families were discovered combining morphological and molecular methods (Ayala et al. 2016). In the southeastern Gulf of California, 47 novel species were found after analyzing ichthyoplankton samples using molecular techniques (Ahern et al. 2018). Likewise, in the central Red Sea, 62 new larval fish families were discovered using a combination of morphological and DNA barcoding approaches (Isari et al. 2017).

In tropical Indian Ocean regions, only few larval identification studies have been described, mostly based on morphological features. Hence, these areas could benefit enormously 
of the advantages of molecular methods to identify ichthyoplankton, combined with morphology (Leis 2015). As DNA barcode-based studies rely on the information provided in databases, expansion of these by submission of DNA sequences is invaluable. Indeed, a study using DNA barcoding found an underestimation of species diversity from both sides of the Indian Ocean (Zemlak et al. 2009). This has been also observed in other studies where 115 adult fish species, including members of the commercially important families Scombridae, Carangidae, Lutjanidae, and Clupeidae, were targeted using molecular methods (Lakra et al. 2011). Taxonomic studies combining morphological and molecular methods on larval and adult fish from the Indian Ocean are scarce (Zemlak et al. 2009; Lakra et al. 2011; Collet et al. 2017). In addition, knowledge concerning the diversity of larval fish in Sri Lankan waters is almost lacking. The adult diversity, however, is known to some extent, mainly based on morphological studies. Recent work exhibited high species diversity in the waters around Sri Lanka and adjacent areas (Joshi et al. 2016; Krakstad et al. 2018). During the same survey as this study, Krakstad et al. (2018) recorded more than 400 species and 100 families of adult fish. Bottom trawl catches from their study showed the South East region to have the highest diversity as compared to the other regions. They further revealed that demersal fish families, Leiognathidae, Diodontidae, Acanthuridae, and Carangidae, dominated in the inner shelf area, whereas in the outer shelf areas, Lutjanidae, Lethrinidae, Carangidae, and Serranidae were abundant.

In the Indian Ocean, monsoon winds play an important role generating currents, waves, variations in sea surface temperature, and other abiotic factors which influence fish behavior, seasonality, distribution, and abundance (Subarna 2018). This can be especially observed during southwest monsoon period, where a distinct pattern in physical properties and productivity characteristics clearly distinguish the eastern and western regions (de Vos et al. 2014; Krakstad et al. 2018). The sea surface temperature (SST) distribution demarcates the survey area into two main distinct zones, a warmer east and a cooler west (Krakstad et al. 2018). Their investigations showed that salinity contrasted the SST with east areas having comparatively lower values (influence from river inflow from the Bay of Bengal) than waters west of Sri Lanka. Furthermore, the east coast is less productive than the west (Yapa 2000; Krakstad et al. 2018).

In the present paper, the larval fish species diversity and composition in the Indian Ocean were mapped for the first time using both molecular and traditional (based on morphological and meristic features) approaches in parallel. We hypothesized that (i) the abiotic (temperature, salinity, and oxygen) and biotic (Chlorophyll $a$, zooplankton) differences in the east and west regions play an important role in shaping overall diversity and abundance patterns of larval fish and (ii) shallow and off shelf waters are important in structuring demersal, pelagic, and mesopelagic larval fish families. The east region is a combination of polygons North East (NE), Central East (CE), and South East (SE), while the west region is the remaining survey area consisting of strata North West (NW), South West (SW), and South (S) (Fig. 1 and Krakstad et al. 2018).

\section{Materials and methods}

\section{Study site and sample collection}

The ecosystem survey on the R/V Dr. Fridtjof Nansen in Sri Lankan waters (located between latitudes $5^{\circ} 24^{\prime}$ and $10^{\circ} 23^{\prime} \mathrm{N}$ and longitudes $78^{\circ} 55^{\prime}$ and $82^{\circ} 25^{\prime} \mathrm{E}$ ) was conducted from 24 June to 16 July 2018 . The time frame overlapped with the southwest monsoon period (de Vos et al. 2014). The geographic area investigated was subdivided into six polygons as NE $\left(18,071 \mathrm{~km}^{2}\right), \mathrm{CE}\left(18,896 \mathrm{~km}^{2}\right)$, SE $\left(28,780 \mathrm{~km}^{2}\right), \mathrm{S}$ $\left(16,231 \mathrm{~km}^{2}\right), \mathrm{SW}\left(33,746 \mathrm{~km}^{2}\right)$, and NW $\left(18,397 \mathrm{~km}^{2}\right)$ (Fig. 1). These polygons were predefined for management purposes based on previous oceanographical studies (Krakstad et al. 2018). There is a relatively narrow continental shelf and a steep slope in most regions off Sri Lanka with $500 \mathrm{~m}$ depth contour delineating the shelf and deep waters.

A shipboard conductivity, temperature, and depth profiler (Sea Bird 911 CTD), with an oxygen sensor and a fluorometer attached to a 12-bottle rosette system, was used to measure vertical distributions of temperature, salinity, and oxygen in the waters around Sri Lanka (Krakstad et al. 2018). Zooplankton was collected using a WP2 ring net with a diameter of $0.56 \mathrm{~m}$ and equipped with a $180 \mu \mathrm{m}$ mesh size. After retrieval, the WP2 net sample collected at the cod end bucket was transferred into a Motoda splitter for dividing into equal halves. One-half was preserved in $4 \%$ formaldehyde for species composition analyses at the laboratory. The other half was used for obtaining size fraction information on zooplankton biomass (Krakstad et al. 2018). The total biomass ( $\mathrm{g} \mathrm{m}^{-2}$ dry weight, at $0-30 \mathrm{~m}$ ) corresponding to larval fish stations was used in this study (Fig. 1).

Larval fish were collected using a Hydro-Bios Multinet Mammoth $\left(1 \mathrm{~m}^{2}, 300 \mu \mathrm{m}\right.$, serial number 2000218) single net at different stations (Fig. 1). The net hauls were taken obliquely from $\sim 25$ to $0 \mathrm{~m}$ at stations with a $30 \mathrm{~m}$ bottom depth and $\sim 100$ to $0 \mathrm{~m}$ at stations with a bottom depth > $100 \mathrm{~m}$ using a single net. The net was gently rinsed, and the sample was collected from the cod end. Visible larval fish were removed from the total sample, photographed (Leica M80 10,450,167 stereomicroscope and a Nikon D610 DSLR camera) and transferred individually to Eppendorf tubes containing $96 \%$ ethanol. The rest of the Multinet Mammoth sample was split into two halves, using a Motoda 
Fig. 1 Locations of Hydro-Bios Multinet Mammoth stations during the survey 24 June to 16 July 2018 around Sri Lanka. The survey region is divided into 6 polygons as shown in the map: North East (NE), Central East (CE), South East (SE), South (S), South West (SW), and North West (NW). Red filled circles indicate Multinet Mammoth stations

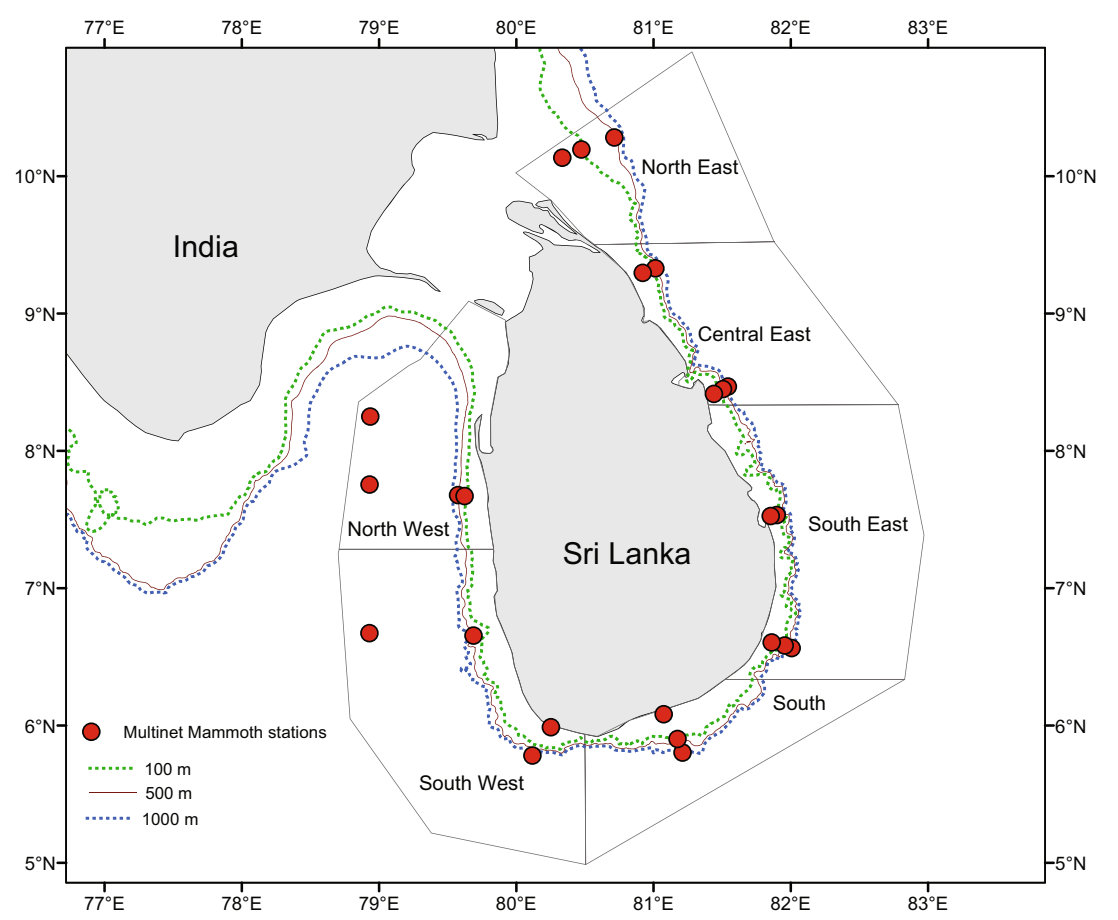

splitter. One-half was preserved in $4 \%$ formaldehyde buffered with borax, and the other half in $96 \%$ ethanol. The half of the samples in formaldehyde were used for morphological taxonomic identification, and rest of the half samples in ethanol were used for DNA barcoding. The volume of the water filtered was obtained from the two flowmeters attached to the mouth of the Multinet Mammoth frame. Abundance (No. $\mathrm{m}^{-3}$ ) was calculated dividing the total number of larvae by the volume filtered.

\section{Morphological and molecular taxonomic methods}

Individual fish larvae were sorted and identified to lowest possible taxonomic level based on the morphological characteristics (body form, the pigmentation pattern (mostly on fresh samples on board), and meristic and morphometric characteristics) using available keys by Leis and Carson-Ewart (2004) and Rodriguez et al. (2017). Total length of individual larval specimens was measured, and the development stages (preflexion, flexion, and postflexion) of majority of dominant larvae were determined. DNA was isolated using HotSHOT, a method widely used for DNA barcoding (Montero-Pau et al. 2008). Briefly, approximately $0.4 \mathrm{~cm}$ of the larval tissue was added into $0.2 \mathrm{~mL}$ tubes containing $50 \mu \mathrm{L}$ of the alkaline lysis buffer $(\mathrm{NaOH} 25 \mathrm{mM}$, disodium EDTA $0.2 \mathrm{mM}, \mathrm{pH} 8.0$ ) and incubated for $30 \mathrm{~min}$ at $95{ }^{\circ} \mathrm{C}$. Afterwards, $50 \mu \mathrm{L}$ of the neutralizing solution (Tris- $\mathrm{HCl}$ $40 \mathrm{mM}, \mathrm{pH}$ 5) were added to the mixture. Upon DNA isolation, PCR amplification targeting the mitochondrial COI gene was performed following the methods described in Mateos-Rivera et al. (2020). We refer to Table S1 for details on PCR amplification, purification, and sequencing. The threshold value ( $>97 \%$ ) used to assign the larval fish sequences to species was chosen following previous studies (Ahern et al. 2018; Burrows et al. 2019). Sequences were uploaded to the GenBank under different accession numbers (Table S2).

\section{Data analysis}

\section{Fish diversity}

Fish family diversity and evenness were calculated for each sampling station and study region using the Shannon-Weaver diversity index (1; Shannon and Weaver 1949), where $S$ is the total number of the species, and $p i$ is the relative abundance of each species (i.e., the numerical proportion of each species relative to total number of individuals in the community).

$H^{\prime}=-\sum_{i=1}^{S}(p i \ln p i)$

Pielou's evenness index, $J$ ' (2; Pielou 1975) was used to study the equality of families for each station and study region, where $H^{\prime}$ is the calculated Shannon-Weaver diversity index value and $S$ is the total number of species.

$J^{\prime}=\frac{H^{\prime}}{\ln S}$ 


\section{Spatial patterns in abiotic and biotic variables}

A nonparametric Kruskal-Wallis test was performed to compare the medians of temperature, salinity, and oxygen. In addition, a principal component analysis (PCA) (Legendre and Legendre 2012) was used to explore how polygons were related to the biotic (chlorophyll $a$, zooplankton biomass, larval fish abundance of demersal, pelagic, mesopelagic, and bathypelagic categories based on habitat criteria defined by associted references in Table 1) and abiotic (temperature, salinity, oxygen, and bottom depth) variables. The PCA was performed with the software "R" v. 3.5.1 (R Core Team 2018) and applying the function "prcomp". As the variables were on different scales, PCA analysis was run on the correlation matrix, which is equivalent to standardizing the variables to mean zero and standard deviation one.

\section{Results}

\section{Temperature, salinity, and oxygen}

A distinct pattern in Sri Lankan waters was observed when comparing temperature, salinity, and oxygen conditions (Fig. 2). In the east region the water was warmer ( $\sim 29$ vs. $27^{\circ} \mathrm{C}$ ) and slightly less saline ( $\sim 34$ vs. 35 ) compared to west (Fig. 2a, b). The south region seems to be a transition zone, with somewhat lower temperatures $\left(26.5^{\circ} \mathrm{C}\right)$ than both east and west and moderate salinty values (34.5). Oxygen levels were more or less constant in the upper layers $\left(\sim 4.5 \mathrm{ml} \mathrm{L}^{-1}\right)$ in the east while it was lower and more variable $\left(3.4-4.4 \mathrm{ml} \mathrm{L}^{-1}\right)$ in the west (Fig. 2c). This regional difference between east (NE, CE, and SE polygons) and west (S, SW, and NW) regions is supported by the Kruskal-Wallis test, where the median of temperature, salinity, and oxygen from the 6 polygons were significanatly different ( $\mathrm{p} \leq 0.0001$, Table S3). Furthermore, there was an increase in salinity and oxygen in the west when moving from South polygon towards the North West polygon, while the eastern regions investigated were more uniform (Fig. 2).

\section{Dominant pelagic, mesopelagic, and demersal species}

The most abundant larval species were categorized based on their adult habitats, i.e., pelagic, mesopelagic, and demersal. Among the identified pelagic species, Selar crumenophthalmus, Dipterygonotus balteatus, Decapterus russelli, Sardinella spp. (S. albella, S. gibbosa, and S. sindensis), and anchovy Encrasicholina punctifer, dominated the larval fish catches (Table 1, Figs. 3a, 4). The carangid S. crumenophthalmus was observed in Central East, South East, South West, and North West polygons (Fig. 4c). The mottled fusilier D. balteatus was mainly distributed in South East and offshore waters of North West polygons (Fig. 4e). Another carangid D. russelli was abundant in the Central East (Fig. 4d), South East and South West polygons while all sardines (Sardinella spp.) and anchovy (E. punctifer) were concentrated in the North West (Fig. 3a).

Deep sea mesopelagic and bathypelagic species were dominated by drift fishes (Nomeidae), myctophids (Myctophidae), deep sea smelts (Bathylagidae), and codlets (Bregmacerotidae). Cubiceps pauciradiatus was the most abundant deep sea species in the larval catches being mainly distributed in the North East, Central East, South West, and North West regions (Fig. 3b). Among Myctophids, Benthosema fibulatum and Diaphus garmani were dominant (Table 1, Fig. 3b). Bathylagoides argyrogaster was present in the South East, South West, and North West with highest abundance in the South West. The codlets Bregmaceros spp. were commonly found in all regions with varying abundance except for South East. Although the sequence identity for Bregmaceros spp. was low (Table S4), the genus was confirmed by their morphological characteristics (Fig. 5h).

Demersal fish species were mainly dominated by Halichoeres zeylonicus, Amblyeleotris wheeleri, Lutjanus spp. (L. fulviflamma, L. johnii, L. lutjanus, L. notatus, and L. quinquelineatus), Upeneus spp. (U. guttatus, $U$. margarethae, and $U$. randalli), and Nemipterus spp. (N. furcosus and N. randalli) (Table 1, Figs. 3c, 4). The gobies (Gobiidae) consisted of four species, i.e., A. wheeleri, Oxyurichthys papuensis, Valenciennea puellaris, and Valenciennea wardii (Table 1) which showed a similar distribution pattern as wrasses. Among gobies, A. wheeleri was the dominant species and restricted to the South East shelf (Fig. 3c). Most snappers including Lutjanus spp. (Lutjanidae) were concentrated in the South East. Four species of threadfin breams (Nemipteridae) were recorded in coastal waters of South East, and in offshore waters in South West and North West polygons. The Goldstripe wrasse, H. zeylonicus (Labridae) was abundant in the Central East, South East, South, and South West polygons (Fig. 4a). Another wrasse, Oxycheilinus spp., was found in the Central East and South polygons with low abundance and all 5 individuals belonging to this genus were assigned to a single OTU, with low sequence identity (Table S4). This genus was also confirmed through their morphological features (Fig. 5g).

\section{Larval tuna composition and distribution}

Five larval tuna species were recorded in the current survey, Auxis rochei, Auxis thazard, Euthynnus affinis, Katsuwonus pelamis, and Thunnus albacares (Table 1, Figs. 5b, 5e and 6). Among these, the Bullet tuna A. rochei was restricted to the Central East shelf region (Fig. 6). The Frigate tuna A. thazard was distributed in the South East, South and North West, whereas E. affinis was distributed in South East shelf and South shelf region (Figs. 5c, e). The Skipjack tuna K. pelamis dominated in the North East and Central East. 
Table 1 Larval fish identified to species level (>97\% sequence identity) in this study. Number of individuals (No.) and regions where individuals were found are given in parenthesis. Habitat of the adults and occurrence in the Indian Ocean (IO) were obtained from literature. North East (NE), Central East (CE), South East (SE), South (S), South West (SW), North West (NW)

\begin{tabular}{|c|c|c|c|c|}
\hline Species & Common name & No. \& Region & Habitat & References \\
\hline Acanthurus mata & Elongate surgeonfish & $1(\mathrm{SW})$ & Reef associated & De Bruin et al. 1994, Rajasuriya 2013 \\
\hline Amblyeleotris wheeleri & Gorgeous prawn-goby & 4 (SE) & Benthic/reef associated & $\begin{array}{l}\text { Kumara and Dalpathadu 2012, Joshi et al. } \\
2016\end{array}$ \\
\hline Apogon crassiceps & Transparent cardinalfish & $1(\mathrm{CE})$ & Reef associated & Froese and Pauly 2019 \\
\hline Auxis rochei & Bullet tuna & $2(\mathrm{CE})$ & Pelagic/neritic & De Bruin et al. 1994 \\
\hline Auxis thazard & Frigate tuna & $8(\mathrm{SE} / \mathrm{S} / \mathrm{NW})$ & Pelagic/neritic & De Bruin et al. 1994 \\
\hline Bathylagoides argyrogaster ${ }^{*}$ & Silver deepsea smelt & 4 (SE/SW/NW) & Mesopelagic & $\begin{array}{l}\text { Gloerfelt-Tarp T, Kailola PJ 1984, Cohen } \\
\text { DM } 1990\end{array}$ \\
\hline Benthosema fibulatum & Spinycheek lanternfish & 4 (NE/CE/SE) & $\begin{array}{l}\text { Benthopelagic and } \\
\text { mesopelagic }\end{array}$ & $\begin{array}{l}\text { Dalpadado and Gjosaeter. 1993, Sebastine } \\
\text { et al. } 2013\end{array}$ \\
\hline Benthosema pterotum & Skinnycheek lanternfish & $1(\mathrm{SE})$ & $\begin{array}{l}\text { Benthopelagic and } \\
\text { mesopelagic }\end{array}$ & $\begin{array}{l}\text { Dalpadado and Gjosaeter 1993, Valinassab } \\
\text { et al. } 2007\end{array}$ \\
\hline Caesio caerulaurea & Blue and gold fusilier & $2(\mathrm{CE})$ & Reef associated & De Bruin et al. 1994, Rajasuriya 2014 \\
\hline Callionymus simplicicornis ${ }^{* *}$ & Simple-spined dragonet & $1(\mathrm{SE})$ & Benthic/reef associated & No records from IO \\
\hline Caranx melampygus & Bluefin trevally & $1(\mathrm{SE})$ & Demersal/reef associated & De Bruin et al. 1994 \\
\hline Ceratias holboelli $^{*}$ & $\begin{array}{l}\text { Kroyer's deep-sea angler } \\
\text { fish }\end{array}$ & $1(\mathrm{NE})$ & Mesopelagic and bathypelagic & Munroe et al. 2015 \\
\hline Ceratoscopelus warmingii & Warming's lantern fish & $1(\mathrm{NE})$ & Mesopelagic & Dalpadado and Gjosaeter 1993 \\
\hline Chanos chanos & Milkfish & 1 (SE) & Benthopelagic/amphidromous & De Bruin et al. 1994 \\
\hline Cheilopogon furcatus & Spotfin flyingfish & $1(\mathrm{NW})$ & Pelagic/neritic/oceanic & De Bruin et al. 1994 \\
\hline Chirocentrus dorab & Dorab wolf-hering & $1(\mathrm{NW})$ & Pelagic & De Bruin et al. 1994 \\
\hline Cirrhilabrus rubeus & $\begin{array}{l}\text { Ruby longfin fairy } \\
\text { wrasse }\end{array}$ & $1(\mathrm{SE})$ & Reef associated & Victor 2016 \\
\hline Coryphaena hippurus & Common dolphinfish & $1(\mathrm{NW})$ & Pelagic & De Bruin et al. 1994 \\
\hline Crossorhombus azureus & Blue flounder & $2(\mathrm{SW} / \mathrm{NW})$ & Benthic & $\begin{array}{l}\text { De Bruin et al. 1994, Nair and } \\
\text { Gopalakrishnan } 2015\end{array}$ \\
\hline Cubiceps pauciradiatus & Bigeye cigarfish & $\begin{array}{l}15 \text { (NE/CE/SW/- } \\
\text { NW) }\end{array}$ & Epipelagic-mesopelagic & Potier et al. 2008 \\
\hline Cynoglossus arel & Largescale tonguesole & $1(\mathrm{SW})$ & Demersal & De Bruin et al. 1994 \\
\hline Decapterus macrosoma & Shortfin scad & $1(\mathrm{SW})$ & Pelagic, Oceanic and Neritic & De Bruin et al. 1994 \\
\hline Decapterus russelli & Indian scad & $6(\mathrm{CE} / \mathrm{SE} / \mathrm{SW})$ & Pelagic, oceanic, and Neritic & De Bruin et al. 1994 \\
\hline Diaphus garmani & Garman's lanternfish & $8(\mathrm{~S} / \mathrm{SW})$ & $\begin{array}{l}\text { Benthopelagic and } \\
\text { mesopelagic }\end{array}$ & Dalpadado and Gjosaeter 1993 \\
\hline Diaphus thiollierei & Thiolliere's lanternfish & $3(\mathrm{CE})$ & Mesopelagic & Dalpadado and Gjosaeter 1993 \\
\hline Diodon holocanthus & $\begin{array}{l}\text { Longspined } \\
\text { porcupinefish }\end{array}$ & 1 (SE) & Benthopelagic/reef associated & De Bruin et al. 1994 \\
\hline Diodon liturosus & $\begin{array}{l}\text { Black-blotched } \\
\text { porcupinefish }\end{array}$ & $1(\mathrm{SE})$ & Benthopelagic/reef associated & Rajasuriya 2013 \\
\hline Dipterygonotus balteatus & Mottled fusilier & $12(\mathrm{SE} / \mathrm{NW})$ & $\begin{array}{l}\text { Reef } \\
\quad \text { associated/pelagic/neritic }\end{array}$ & De Bruin et al. 1994, Rajasuriya 2014 \\
\hline Encrasicholina heteroloba & Shorthead anchovy & $1(\mathrm{SW})$ & Pelagic/neritic & De Bruin et al. 1994 \\
\hline Encrasicholina punctifer & Buccaneer anchovy & $3(\mathrm{NW})$ & Pelagic/neritic & De Bruin et al. 1994 \\
\hline Equulites sp. & Pony fish & $2(\mathrm{NW})$ & Demersl & De Bruin et al. 1994 \\
\hline Euthynnus affinis & Kawakawa & $4(\mathrm{SE} / \mathrm{S})$ & Pelagic/neritic & De Bruin et al. 1994 \\
\hline Gazza minuta & Toothpony & $1(\mathrm{SE})$ & Demersal & De Bruin et al. 1994 \\
\hline Gempylus serpens & Snake mackerel & $2(\mathrm{CE})$ & Epipelagic and mesopelagic & De Bruin et al. 1994 \\
\hline Halichoeres zeylonicus & $\begin{array}{l}\text { Goldstripe/Ceylon } \\
\text { wrasse }\end{array}$ & $5(\mathrm{CE} / \mathrm{SE} / \mathrm{S})$ & Reef associated & De Bruin et al. 1994, Rajasuriya 2014 \\
\hline Katsuwonus pelamis & Skipjack tuna & $6(\mathrm{NE} / \mathrm{CE})$ & Pelagic/oceanic & De Bruin et al. 1994 \\
\hline Lagocephalus guentheri* & Diamondback puffer & $1(\mathrm{SE})$ & Demersal & Mishra et al. 2018 \\
\hline $\begin{array}{l}\text { Leiognathus lineolatus/Equulites } \\
\text { lineolatus }\end{array}$ & Ornate ponyfish & $2(\mathrm{SW} / \mathrm{NW})$ & Demersal & De Bruin et al. 1994 \\
\hline Lethrinus olivaceus & Longface emperor & $1(\mathrm{SE})$ & Demersal & De Bruin et al. 1994 \\
\hline $\begin{array}{l}\text { Liza macrolepis/Planiliza } \\
\quad \text { macrolepis }\end{array}$ & Largescale mullet & $3(\mathrm{~S} / \mathrm{SW})$ & $\begin{array}{l}\text { Benthopelagic/neritic and } \\
\text { estuarine }\end{array}$ & De Bruin et al. 1994 \\
\hline Liza tade/Chelon planiceps & Tade mullet & $1(\mathrm{NE})$ & $\begin{array}{l}\text { Benthopelagic/neritic and } \\
\text { estuarine }\end{array}$ & De Bruin et al. 1994 \\
\hline Lutjanus fulviflamma & Dory snapper & $1(\mathrm{CE})$ & Reef associated & Rajasuriya 2014 \\
\hline Lutjanus johnii & John's snapper & 3 (SE) & $\begin{array}{l}\text { Reef } \\
\quad \text { associated/oceanodromous }\end{array}$ & De Bruin et al. 1994 \\
\hline Lutjanus lutjanus & Bigeye snapper & $1(\mathrm{SE})$ & Reef associated & De Bruin et al. 1994, Rajasuriya 2014 \\
\hline Lutjanus notatus ${ }^{*}$ & Bluestriped snapper & $2(\mathrm{SE})$ & Reef associated & Velamala et al. 2017 \\
\hline
\end{tabular}


Table 1 (continued)

\begin{tabular}{|c|c|c|c|c|}
\hline Species & Common name & No. \& Region & Habitat & References \\
\hline Lutjanus quinquelineatus & Five-lined snapper & $1(\mathrm{NW})$ & Reef associated & De Bruin et al. 1994, Rajasuriya 2013 \\
\hline Mene maculata & Moonfish & $3(\mathrm{SW} / \mathrm{NW})$ & Semi-demersal & De Bruin et al. 1994 \\
\hline Mugil cephalus & Flathead mullet & $2(\mathrm{SW})$ & $\begin{array}{l}\text { Benthopelagic/neritic and } \\
\text { estuarine }\end{array}$ & De Bruin et al. 1994, Rajasuriya 2013 \\
\hline Nemipterus furcosus & Forktail threadfin bream & $2(\mathrm{SE} / \mathrm{SW})$ & Demersal & De Bruin et al. 1994 \\
\hline Nemipterus randalli & $\begin{array}{l}\text { Randall's threadfin } \\
\text { bream }\end{array}$ & $1(\mathrm{NW})$ & Demersal & De Bruin et al. 1994 \\
\hline Notolychnus valdiviae & Topside lampfish & $1(\mathrm{NW})$ & Mesopelagic & Dalpadado and Gjosaeter 1993 \\
\hline Oxyporhamphus micropterus & Bigwing halfbeak & $1(\mathrm{NW})$ & Pelagic/oceanic & Nair and Kumar 2018 \\
\hline Oxyurichthys papuensis & Frogface goby & $1(\mathrm{SE})$ & Benthic & Pezold and Larson 2015 \\
\hline Parablennius thysanius & Tasseled blenny & $1(\mathrm{NW})$ & Benthic/reef associated & Wlliams 2014 \\
\hline Planiliza subviridis & Greenback mullet & $1(\mathrm{NW})$ & $\begin{array}{l}\text { Benthopelagic/neritic and } \\
\text { estuarine }\end{array}$ & De Bruin et al. 1994 \\
\hline Pomacentrus caeruleus & Caerulean damsel & $2(\mathrm{NE} / \mathrm{SE})$ & Reef associated & Kumara and Dalpathadu 2012 \\
\hline Pseudanthias marcia* & Marcia's anthias & $3(\mathrm{NE} / \mathrm{CE} / \mathrm{S})$ & Reef associated & Nair 2008 \\
\hline Pterocaesio tessellata & One-stripe fusilier & $2(\mathrm{CE} / \mathrm{SE})$ & Reef associated & De Bruin et al. 1994 \\
\hline Rastrelliger kanagurta & Indian mackerel & $2(\mathrm{SW})$ & Pelagic/neritic & De Bruin et al. 1994 \\
\hline Rhabdamia gracilis & Luminous cardinalfish & $1(\mathrm{CE})$ & Reef associated & Froese and Pauly 2019 \\
\hline Sardinella albella & White sardinella & $1(\mathrm{NW})$ & Pelagic/neritic & De Bruin et al. 1994 \\
\hline Sardinella gibbosa & Goldstripe sardinella & $2(\mathrm{NW})$ & Pelagic/neritic & De Bruin et al. 1994 \\
\hline Sardinella sindensis & Sind sardinella & $1(\mathrm{NW})$ & Pelagic/neritic & Karunasinghe et al. 2000, Di Dario 2018 \\
\hline Sargocentron punctatissimum & Speckled squirrelfish & $1(\mathrm{SE})$ & Reef associated & De Bruin et al. 1994 \\
\hline Scolopsis bimaculata & $\begin{array}{l}\text { Thumbprint monocle } \\
\text { bream }\end{array}$ & $1(\mathrm{SE})$ & Demersal & De Bruin et al. 1994, Rajasuriya 2014 \\
\hline Scolopsis xenochrous & $\begin{array}{l}\text { Oblique-barred monocle } \\
\text { bream }\end{array}$ & $1(\mathrm{SE})$ & Demersal & Rajasuriya 2014 \\
\hline Selar crumenophthalmus & Bigeye scad & 19 (SE/SW/NW) & $\begin{array}{l}\text { Semidemersal/reef } \\
\text { associated/neritic }\end{array}$ & De Bruin et al. 1994 \\
\hline Sphyraena jello & Pickhandle barracuda & $1(\mathrm{SW})$ & Pelagic/neritic & De Bruin et al. 1994 \\
\hline Sufflamen chrysopterum & Halfmoon triggerfish & $1(\mathrm{SE})$ & Reef associated & De Bruin et al. 1994 \\
\hline Synanceia verrucosa & Stonefish & 1 (CE) & Benthic/reef associated & Motomura et al. 2018 \\
\hline Terapon jarbua & Jarbua terapon & $1(\mathrm{SW})$ & Benthopelagic/estuarine & De Bruin et al. 1994 \\
\hline Thunnus albacares & Yellowfin tuna & $1(\mathrm{CE})$ & Pelagic/neritic & De Bruin et al. 1994 \\
\hline Trachinocephalus myops & Snakefish & $2(\mathrm{NW})$ & Benthic/reef associated & De Bruin et al. 1994 \\
\hline Trichiurus auriga & Pearly hairtail & $2(\mathrm{NW})$ & Benthopelagic/mesopelagic & Ganga et al. 2015 \\
\hline Trichonotus setiger & Spotted sand-diver & 1 (SE) & Benthic & De Bruin et al. 1994 \\
\hline Upeneus guttatus & Two-tone goatfish & $1(\mathrm{SW})$ & Demersal/reef associated & $\begin{array}{l}\text { De Bruin et al. 1994, Uiblein and Heemstra } \\
2010\end{array}$ \\
\hline Upeneus margarethae & Margaretha's goatfish & $3(\mathrm{~S} / \mathrm{NW})$ & Demersal/reef associated & $\begin{array}{l}\text { De Bruin et al. 1994, Uiblein and Heemstra } \\
2010\end{array}$ \\
\hline Upeneus randalli ${ }^{*}$ & & $5(\mathrm{NW})$ & Demersal/reef associated & Uiblein and Heemstra 2010 \\
\hline Valenciennea puellaris & Maiden goby & $1(\mathrm{SE})$ & Benthic & $\begin{array}{l}\text { Long et al. 2010, Hoese and Larson 1994, } \\
\text { Larson 2016a }\end{array}$ \\
\hline Valenciennea wardii ${ }^{*}$ & Ward's sleeper & $1(\mathrm{SW})$ & Benthic & Larson $2016 \mathrm{~b}$ \\
\hline
\end{tabular}

* Species previously not recorded from Sri Lankan waters

** Species not previously recorded from the Indian Ocean

Skipjack tunas were present in both shelf and offshore regions of the North East while concentrated in the shelf region of Central East. The Yellowfin tuna T. albacares was restricted to the Central East deep (Fig. 5d).

\section{New records of occurrences}

The results from DNA barcoding revealed new records of species in the Indian Ocean and Sri Lankan waters (Table 1). To our knowledge, Callionymus simplicicornis (South East shelf) was not previously reported from the Indian Ocean. Several additional species including Bathylagoides argyrogaster
(South East shelf, South West deep, and North West deep), Ceratias holboelli (North East shelf), Lagocephalus guentheri (South East shelf), Lutjanus notatus (South East shelf/deep), Upeneus randalli (North West shelf), Pseudanthias marcia (North East/ Central East/ South deep), and Valenciennea wardii (South West shelf) were not previously recorded from Sri Lankan waters.

\section{Size structure and developmental stage}

The average size (total length) of dominant pelagic larvae, i.e. Selar crumenophthalmus, was larger in the east than the west 

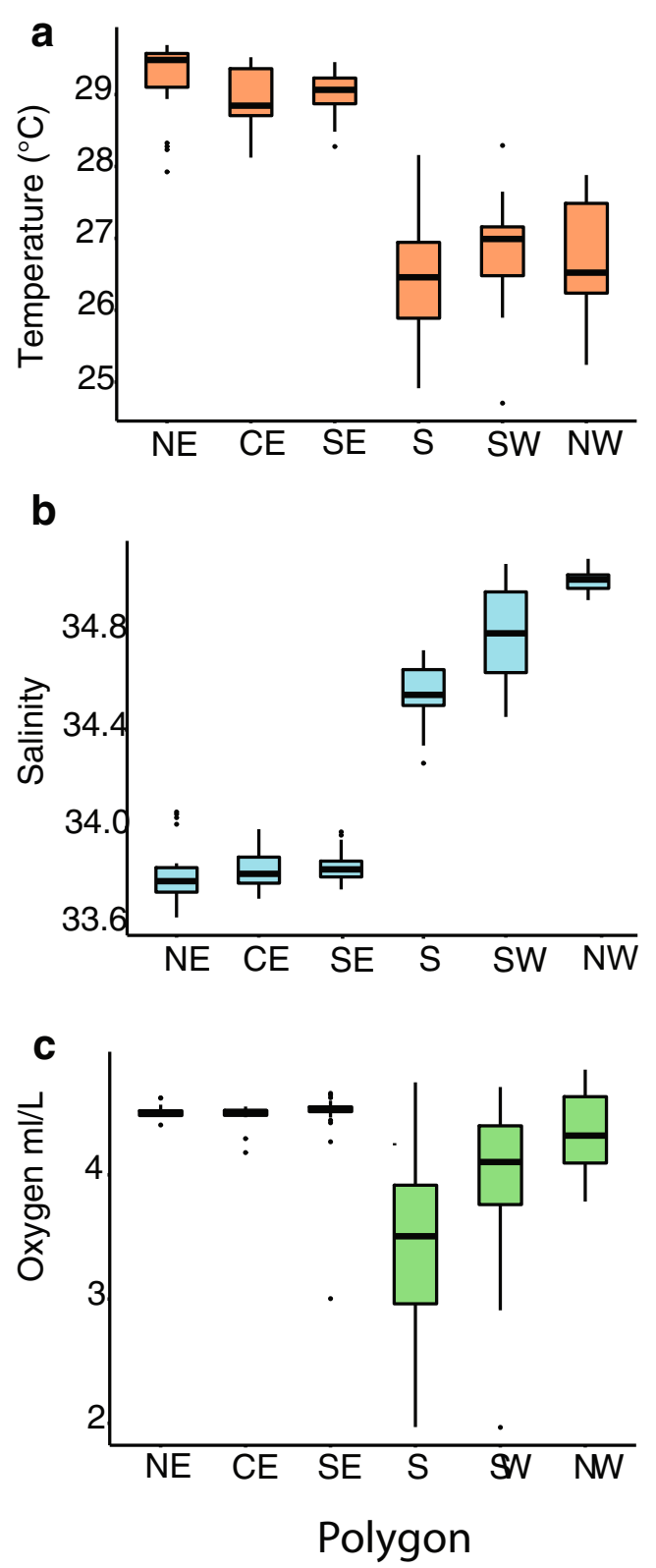

Fig. 2 Box plots of temperature (a), salinity (b), and oxygen (c) at $0-25 \mathrm{~m}$ in the 6 polygons around Sri Lanka: North East (NE), Central East (CE), South East (SE), South (S), South West (SW), and North West (NW). The boxes are divided by the median value and framed by the upper and lower quartiles. The whiskers extend to the highest and lowest observations in each direction. Outliers are shown by separate points

(5.7 $\mathrm{mm}$ and $3.8 \mathrm{~mm}$, respectively). Within pelagic larval fish, for Dipterygonotus balteatus, the average size was ca. $5 \mathrm{~mm}$ in both regions (SD 2.1). The dominant mesopelagic larvae Cubiceps pauciradiatus in both east and west regions had an average length $5.5 \mathrm{~mm}$ (east $\mathrm{SD}=1.5$ and west $\mathrm{SD}=1.2$ ). Most demersal larvae of Lutjanus spp. and Halichoeres zeylonicus recorded in this study were in the late post flexion stage, indicating they are nearing juvenile stage. Lutjanus spp. were in general much larger, ranging in size up to $26 \mathrm{~mm}$, whereas $H$. zeylonicus varied from 7.8 to $13.5 \mathrm{~mm}$.

\section{Family diversity, species composition, and spatial distribution}

Shannon-Weaver diversity index showed a high larval fish family diversity with some variations among stations and regions. The highest larval diversities were observed in the South East $(H=2.96, E=0.72)$, followed by South West $(\mathrm{H}=2.80, \mathrm{E}=0.78)$, North West $(\mathrm{H}=2.78, \mathrm{E}=0.78)$, and North East $(\mathrm{H}=2.50, \mathrm{E}=0.83)$ polygons. The lowest diversity was recorded from the Central East $(\mathrm{H}=1.57, \mathrm{E}=0.5)$ and South $(\mathrm{H}=2.14, \mathrm{E}=0.66)$ polygons.

Among the 551 larval fish analyzed following the molecular approach, 305 individuals were successfully identified (55\%) and divided into two different categories based on sequence identity threshold when compared to the databases (Table 1, Table S4). In total $65 \%$ of the individuals ( 80 species) were identified at $>97 \%$ sequence identity (Table 1), while 35\% (53 operational taxonomic units (OTUs)) were assigned at a lower identity (Table S4). Morphological identification of larval fish was successful to family level for up to $70 \%$ of the total samples.

Overall, Carangidae was the most abundant family among the identified larval fish. It was followed by Myctophidae Lutjanidae, Scombridae, and Caesionidae. Myctophidae and Scombridae with six different species, Lutjanidae with five species, while Caesionidae, Carangidae, Clupeidae, Gobiidae, Mugilidae, and Nemipteridae contained four species (Table 1).

\section{Clustering analysis}

The first two axes of the PCA together explained $\sim 67 \%$ of the variance in the data set across stations (Fig. 7). The PCA analysis showed a clear separation of eastern and western stations along the PC1 axis, which explained most of the variance $(\sim 44 \%)$. Increasing PC1 scores are associated with decreasing salinity and increasing temperature. Hence, we find eastern stations associated with warmer less saline waters on the righthand side of the PCA biplot (Fig. 7, in red), while western stations characterized by cooler and more saline waters with less oxygen and higher productivity are found on the left-hand side of the biplot (Fig. 7, in blue). Mesopelagic larvae loadings are explained by both $\mathrm{PC} 1$ (being associated predominantly in cooler, less oxygenated waters in the west) and PC2 (correlated with depth). The demersal and pelagic larval abundances were associated with PC2 axis (which explains $\sim 23 \%$ of the variance) likely driven by their high 
Fig. 3 Most abundant (No. 1000 $\mathrm{m}^{-3}$ ) larval fish species categorized based on adult habitats: a pelagic, $\mathbf{b}$ mesopelagic, and $\mathbf{c}$ demersal. Note that overlapping stations are placed slightly apart, for better visualization of the species composition. Species are listed in abbreviated form according to descending abundance at sampling locations. Pelagic: Selar crumenophthalmus (Sc),

Decapterus russelli (Dr), Dipterygonotus balteatus (Db), Encrasicholina punctifer (Ep),

Sardinella spp. (Sa); mesopelagic: Cubiceps pauciradiatus $(\mathrm{Cp})$,

Bathylagoides argyrogaster $(\mathrm{Ba})$, Benthosema fibulatum (Bf),

Diaphus garmani (Dg), Bregmaceros spp. (Br); and demersal: Halichoeres zeylonicus (Hz), Amblyeleotris wheeleri (Aw), Lutjanus spp. (Lu), Oxycheilinus spp (Ob), Upeneus spp (Up)
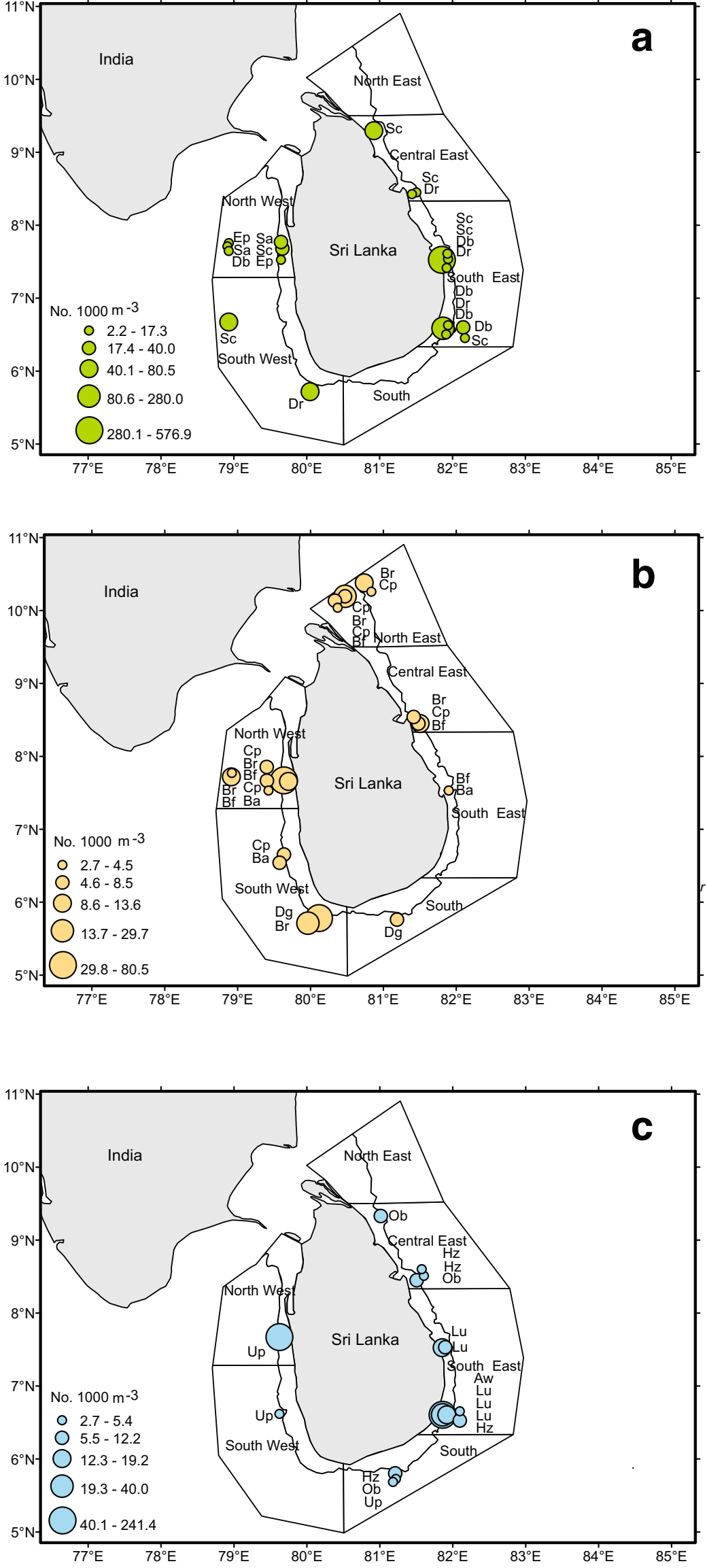
Fig. 4 Images of few abundant fish species recorded during the survey 24 June to 16 July 2018. Their total length (TL) and \% sequence identity (ID) are given: a Halichoeres zeylonicus $(\mathrm{Hz})$ $15 \mathrm{~mm}$ TL, $100 \%$ (ID); b Lutjanus notatus (Ln), $14 \mathrm{~mm}$ TL, $100 \%$ (ID); c Selar crumenophthalmus (Sc), $7.1 \mathrm{~mm}$ TL, $100 \%$ (ID); d Decapterus russelli (Dr), $6.3 \mathrm{~mm}$ TL, 100\% (ID); e Dipterygonotus balteatus (Db), $8.2 \mathrm{~mm}$ TL, $98.2 \%$ (ID); $\mathbf{f}$ Cubiceps pauciradiatus (Cp) $5.8 \mathrm{~mm}$ TL, > 99\% (ID). The images were taken on fresh specimens

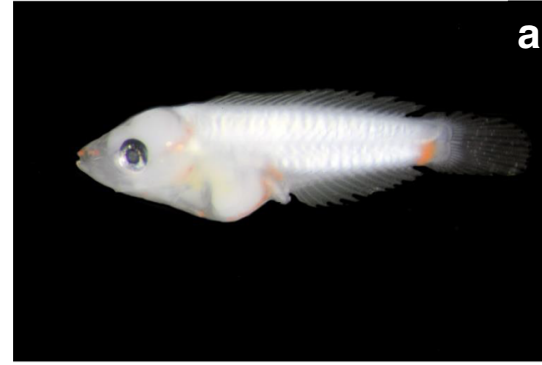

a
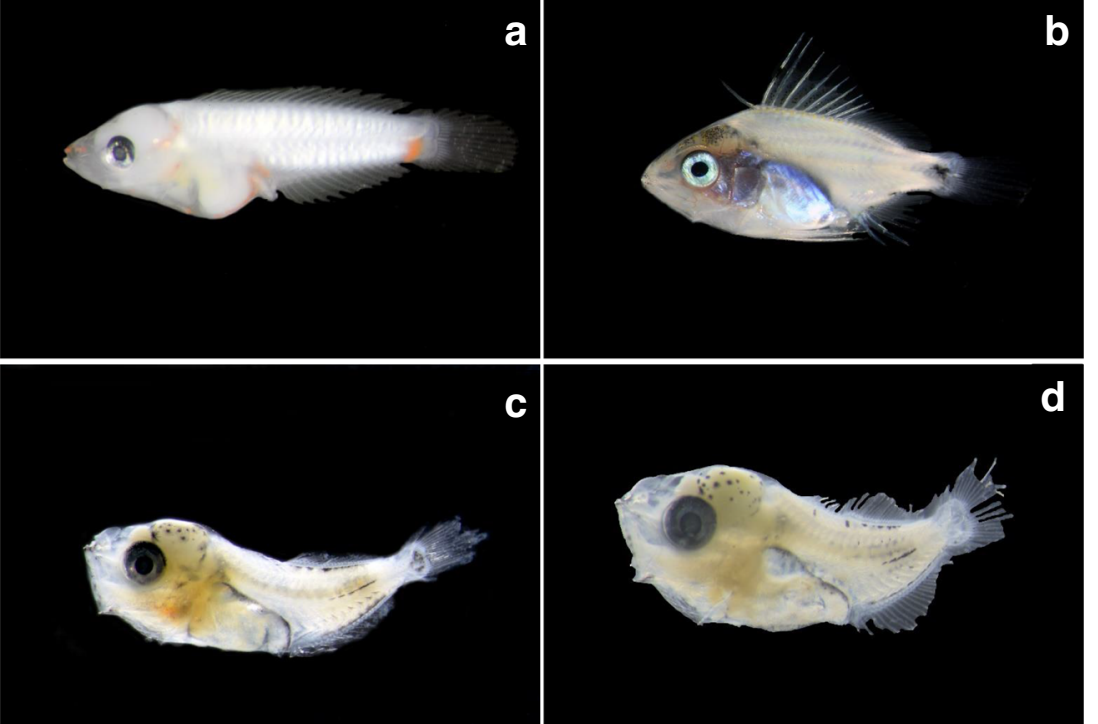

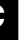

d
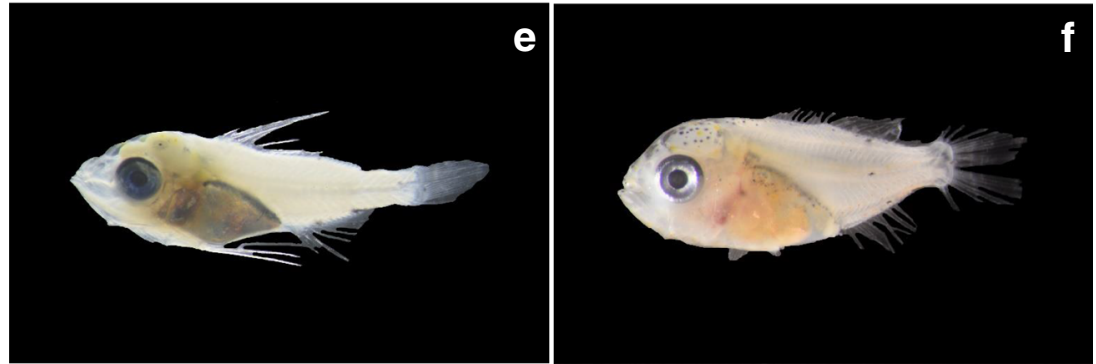

abundances in the shallower South West and South East shelf stations (stations 10,13 and 18).

\section{Discussion}

The current study provides an insight into the larval fish diversity and structuring in a sparsely studied area of the central Indian Ocean. In our study, morphological identification of larval fish was successful up to family level in $70 \%$ of the samples. Visual identification to genus and species levels, however, was only possible for a few species with distinct morphological features or those that are well documented due to their commercial importance such as Mene maculata, Coryphaena hippurus, Auxis thazard, Euthynnus affinis, Katsuwonus pelamis, and Thunnus albacares (see Fig. 5). All the above-mentioned species were cross validated with DNA barcoding.

Molecular barcoding is a powerful tool for identification of larval fish species (Hubert et al. 2015; Ayala et al. 2016; Ahern et al. 2018), and required to thoroughly monitor larval recruitment and year class-strength (Steinke et al. 2016). Combining morphological and DNA barcoding allowed identification of 80 species of larval fish belonging to 69 families in our study, demonstrating a very high diversity in the tropical central Indian Ocean and exceeding previously reported numbers from this region. Morphological approaches alone are much more challenging for accurate identification of larval fish species compared to adults, highlighting the urgent need for using molecular approaches in taxonomic investigations from this region (Collet et al. 2017).

Carangidae was the most abundant family observed. Studies on the reproductive cycles of the two most dominant carangids Selar crumenophthalmus and Decapterus russelli showed a prolonged spawning season from ca. April to November in the south west Indian Ocean and south west coast of India (Balasubramanian and Natarajan 2000; Roos et al. 2007). Although no records of spawning of D. russelli and S. crumenophthalmus on the Sri Lankan shelf are available, our results indicate that their spawning also takes place in Sri Lankan shelf areas.

The mottled fusilier Dipterygonotus balteatus is abundant in the Indian Ocean inhabiting coral reefs as juveniles, whereas adults are captured together with other nearshore pelagic fish such as sardines and anchovies (Carpenter 1988). In our study, this species dominated throughout the coastal waters of South East and offshore waters of North West. Although this species extends its distribution from western Indian Ocean into the western Pacific (Holleman et al. 2013), its spawning sites in the Indian Ocean are unknown (Holleman et al. 2013). 
Fig. 5 Images of fish species and genera with special

morphological characteristics including tuna species recorded during the survey 24 June to 16 July 2018. Their total length (TL) and $\%$ sequence identity (ID) are given: a Mene maculata (Mm), $7 \mathrm{~mm}$ TL, 100\% (ID); b Katsuwonus pelamis (Kp), $9.1 \mathrm{~mm}$ TL, 100\% (ID); c Euthynnus affinis (Ea), $9.2 \mathrm{~mm}$ TL, $100 \%$ (ID); d Thunnus albacares (Ta), $9.0 \mathrm{~mm} \mathrm{TL}$, $99.8 \%$ (ID); e Auxis thazard (At), $8.4 \mathrm{~mm} \mathrm{TL}, 100 \%$ (ID); f Coryphaena hippurus (Ch), $5.2 \mathrm{~mm}$ TL, $99.8 \%$ (ID); $\mathbf{g}$ Oxycheilinus spp. (Ob) $8.5 \mathrm{~mm}$ TL, $93.3 \%$ (ID); h Bregmaceros spp. (Br) $16.8 \mathrm{~mm} \mathrm{TL}, 94 \%$ (ID). The images were taken on fresh specimens

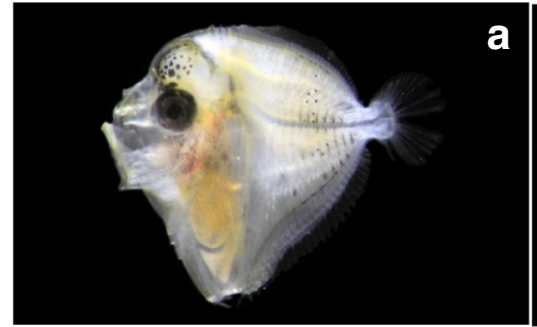

a

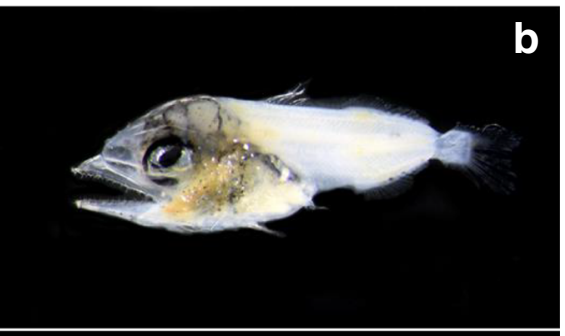

C

d
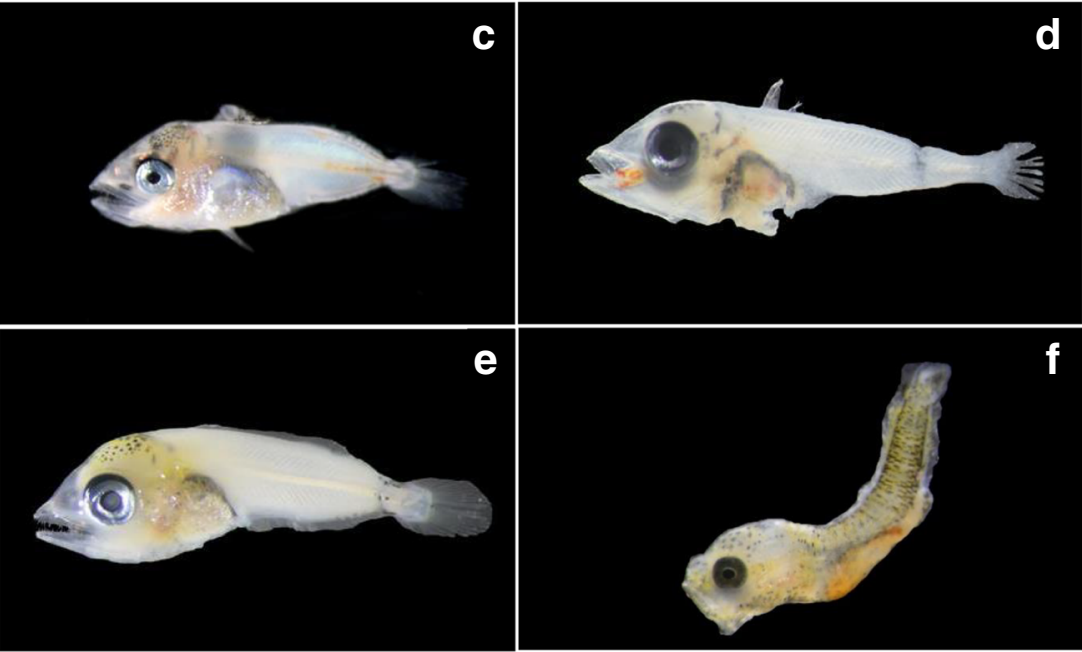

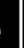

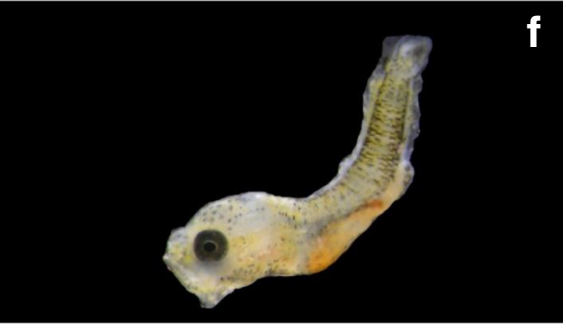

g

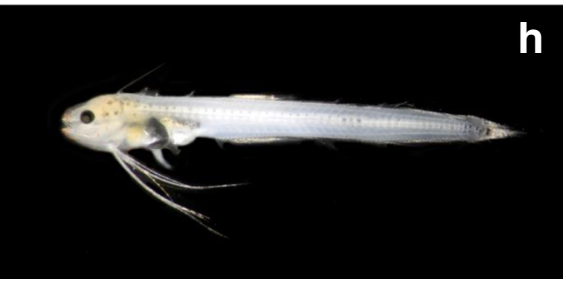

The larger pelagic tunas spawn at sea surface temperatures above $20{ }^{\circ} \mathrm{C}$ (Reglero et al. 2014), suggesting that waters around Sri Lankan are suitable spawning grounds. The spawning of Skipjack (Katsuwonus pelamis) is relatively high in the northern and central Indian Ocean during September through April (Pillai and Silas 1979), while the Yellowfin tuna, Thunnus albacares, spawns off Sri Lanka from April to June (Stequert and Marsac 1989). The smaller Frigate tuna,
Fig. 6 Distribution of most abundant larval tuna species. Note that overlapping stations are placed slightly apart, for better visualization of the species composition. Species are listed in abbreviated form according to descending abundance at sampling location. Katsuwonus pelamis (Kp), Thunnus albacares (Ta), Auxis thazard (At), Auxis rochei (Ar), Euthynnus affinis (Ea)

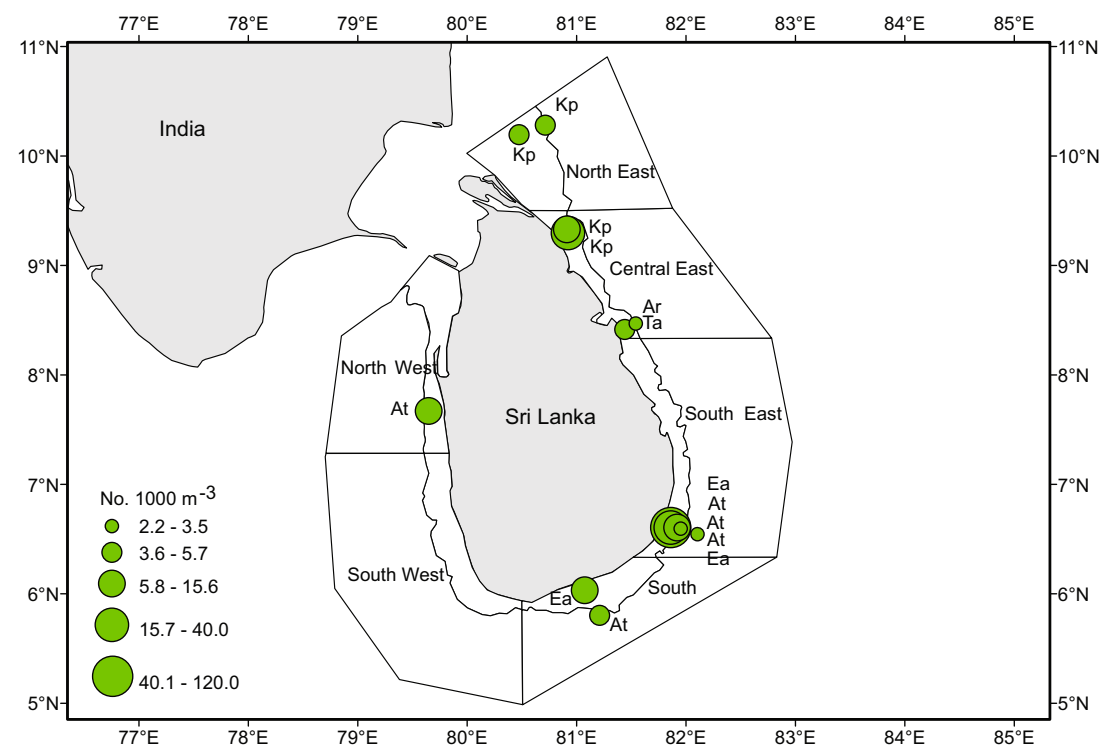




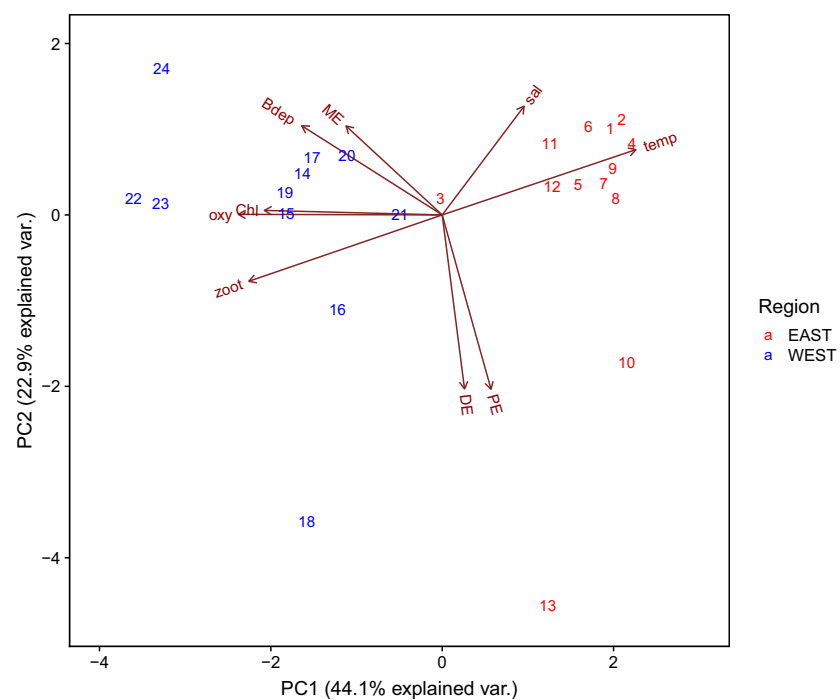

Fig. 7 PCA biplot separating the different stations (1-24). Abiotic variables: temperature (temp), salinity (sal), and oxygen (oxy), bottom depth (Bdep) and biotic variables: chlorophyll $a(\mathrm{Chl})$, zooplankton total biomass (zoot), larval fish abundance for demersal (DE), pelagic (PE), and mesopelagic (ME). See Table S3 for more details on corresponding survey station for each PCA station. The PCA biplot is designated in two colors: left side in "blue" and the right side in "red"

Auxis thazard, spawns in Sri Lankan waters from May to August (Pillai and Satheeshkumar 2012; Herath et al. 2019) overlapping the period of Kawakawa tuna Euthynnus affinis (Pillai and Satheeshkumar 2012). This dominance of larval tuna in coastal regions off Sri Lanka indicates that they are likely drifted with the surface currents (Fig. S1) from spawning sites into nursery areas with good feeding conditions (Krakstad et al. 2018). All fish stocks that live in areas characterized by stable current systems have genetic predispositions to secure their survival within a geographical area (Harden-Jones 1968). The author outlines a life cycle with an upstream spawning area and a downstream nursery area and the general applicability of this theory has been proven for numerous fish stocks in several geographical areas such as in the North Atlantic (ICES 2019) and South African coast bordering both south Atlantic and Indian Ocean (Hutchings et al. 2002). The study by Hutchings et al. (2002) showed that most pelagic and demersal fish species have evolved highly selective reproductive strategies, among other that fish conduct spawning migrations to upstream areas from where the offspring are transported with the currents to suitable nursery areas, such as productive retention and broader shelf areas.

Myctophids are the most diverse and common group in oceanic waters (Lirdwitayaprasit et al. 2008; Ayala et al. 2016; Isari et al. 2017; Beckley et al. 2019). Recent studies highlighted the large contribution of Myctophidae to the mesopelagic vertebrate biomass ( $>50 \%)$ and their importance in the midwater food web (Martin et al. 2018; Batta-Lona et al. 2019 and references therein). Though their high biomass in the Indian Ocean has been known for several decades (FAO
2001), yet until recently there has been comparatively little research activity on the mesopelagic zone (Hildago and Browman 2019). Myctophidae were the second most abundant family in this study associated with high saline, cooler, less oxygenated, deeper waters in the south and west regions (South, South West, and North West). Myctophids are known to exhibit adaptations to oxygen minimum zones (Catul et al. 2011), hence, likely their success in the Indian Ocean. The oxygen minimum layer $(<0.5 \mathrm{ml} / \mathrm{L})$ off Sri Lanka is below $100 \mathrm{~m}$ (Krakstad et al. 2018); hence, the myctophids we observed in our study are likely to experience low oxygen zones. A study by Olivar et al. (2018) showed that the vertical distribution of larval mesopelagic fish varied from 0 to $200 \mathrm{~m}$ in the tropical and equatorial Atlantic depending on the species. Another study in the Kuroshio Current region exhibited larval myctophid species to occupy different depth strata (Sassa et al. 2002). Their study showed that larvae belonging to subfamily Lampanyctinae occupied shallower waters $(0-30 \mathrm{~m})$ than those in the subfamily Myctophinae (50-150 m).

The majority of the demersal larval fish species identified (Lutjanus notatus, L. lutjanus, L. johnii, Halichoeres zeylonicus, Oxycheilinus spp., and Upeneus margarethae) recorded in this study in the South and South East are consistent with rocky reef habitats favorable for adults of these species (Krakstad et al. 2018). The adult habitat and spawning behavior seem to explain the spatial patterns of the demersal larval fish assemblages in Sri Lankan waters. Similar to our findings, Hubert et al. (2015) using molecular tools for ichthyoplankton identification in the Indo-Pacific region revealed that most of the species identified in larval pools reflected the local contribution from the adult pools of demersal fish in the neighboring reefs. Sakaue et al. (2016) also revealed that the larvae of some Lutjanidae species were found near adult spawning aggregations, supporting our observations.

Furthermore, the use of molecular barcoding in this study revealed a new species Callionymus simplicicornis that has not been reported before in the Indian Ocean. The simple-spined dragonet $C$. simplicicornis is found in southeast Asian waters, i.e., Philippine waters, Palau/Belau islands (Fricke 2002). There is a possibility that this species known to occur in the Pacific have entered the Indian Ocean through the "Indonesian Throughflow (ITF)" (Tomczak and Godfrey 1994). According to these authors, water exchange between the Indian Ocean and the Pacific Oceans occurs through the ITF, where water from the Pacific flows into the Indian Ocean, as a narrow band of low salinity water. A branch of the ITF water penetrates the northern Indian Ocean via Somali Current further into the Arabian Sea during boreal summer (Song et al. 2004). During summer monsoon, the Southwest Monsoon Current transfers Arabian Sea waters eastward to offshore areas around the southern tip of India and Sri Lanka (Hood et al. 2017). The simple-spined dragonet is a small demersal reef associated species inhabiting coastal waters. As a non-commercial small 
species and due to the lack of taxonomic studies, these fish could be considered as "overlooked species".

Majority of the larvae were in flexion and post flexion developing stages, indicating that they use the waters around Sri Lanka as nursery grounds. It is likely that some of the larvae especially in early development stages are dispersed by the South Monsoon Current from the west to the eastern regions (Fig. S1). Studies by Beldade et al. (2006) and Borges et al. (2007) on larval fish assemblages in nearshore waters have described that particularly postflexion stages, close to the bottom, are less susceptible to transport hence, contained close to adult habitats. The late post flexion larvae of demersal families in the east region seem to indicate that they remain close to where adult spawning occurs.

In conclusion, our study shows that combined morphological and molecular approaches can reveal vast species diversity. Dominance of several larval fish species in the east during the survey seem to indicate that oligotrophic areas in the east can also sustain high diversity. Findings from this study exhibit that Sri Lankan waters act as valuable nursery grounds for a wide range of fish species.

Supplementary Information The online version contains supplementary material available at https://doi.org/10.1007/s12526-021-01169-w.

Acknowledgments This paper uses data collected through the scientific surveys with the R/V Dr. Fridtjof Nansen as a part of the collaboration between the EAF-Nansen Programme and the Government of Sri Lanka. The EAFNansen Programme is a partnership between the Food and Agriculture Organization of the United Nations (FAO), the Norwegian Agency for Development Cooperation (Norad), the Institute of Marine Research (IMR), Norway, and partner countries and institutes e.g. National Aquatic Resources Research and Development Agency (NARA), Sri Lanka. We thank the EAFNansen Programme and the Government of Sri Lanka for their immense support. Our thanks are also extended to the bilateral project between Sri Lanka and Norway (technical assistance to improve management of the fish resources of Sri Lanka. LKA-3183, LKA-16/0005) for strengthening ichthyoplankton research in this region. The authors are indebted to Dr. Palitha Kithsiri, the Deputy Director General, Research and Development of NARA and Coordinator of EAF-Nansen Programme, Sri Lanka, for providing facilities and encouragement. We are very grateful to the academic editor and the two anonymous referees for their valuable and constructive comments on the manuscript.

Funding Open Access funding provided by the Institute of Marine Research, Norway. This research was funded by EAF Nansen program of Food and Agriculture Organization (FAO), the Norwegian Agency for Development Cooperation (Norad) and the government of Sri Lanka (Grant number 15113-39).

\section{Declarations}

Conflict of interest The authors declare that they have no conflict of interest.

Ethical approval All applicable international, national, and/or institutional guidelines for the care and use of animals were followed by the authors.
Sampling and field studies All necessary permits for sampling and observational field studies have been obtained by the authors from the competent authorities and are mentioned in the acknowledgements.

Data availability All data generated or analyzed during this study are included in this published article.

Author contributions Project coordination PD. In situ data collection and database management MIGR, HBUW, RPPKJ, JOK, PD. DNA barcoding AMR, RSM. Analytical tools and visualization AMR, MIGR, PD. Writing - original draft MIGR, AMR, PD. Writing - review \& editing MIGR, AMR, RSM, HBUW, RPPKJ, JOK, PD.

Open Access This article is licensed under a Creative Commons Attribution 4.0 International License, which permits use, sharing, adaptation, distribution and reproduction in any medium or format, as long as you give appropriate credit to the original author(s) and the source, provide a link to the Creative Commons licence, and indicate if changes were made. The images or other third party material in this article are included in the article's Creative Commons licence, unless indicated otherwise in a credit line to the material. If material is not included in the article's Creative Commons licence and your intended use is not permitted by statutory regulation or exceeds the permitted use, you will need to obtain permission directly from the copyright holder. To view a copy of this licence, visit http://creativecommons.org/licenses/by/4.0/.

\section{References}

Ahern AL, Gómez-Gutiérrez J, Aburto-Oropeza O, Saldierna-Martínez RJ, Johnson AF, Harada AE, add Sánchez-Uvera AR, Erisman B, Castro Arvizú DI, Burton RS (2018) DNA sequencing of fish eggs and larvae reveals high species diversity and seasonal changes in spawning activity in the southeastern Gulf of California. Mar Ecol Prog Ser 592:159-179. https://doi.org/10.3354/meps 12446

Ayala D, Riemann L, Munk P (2016) Species composition and diversity of fish larvae in the Subtropical Convergence Zone of the Sargasso Sea from morphology and DNA barcoding. Fish Oceanogr 25:85-104. https://doi.org/10.1111/fog.12136

Balasubramanian NK, Natarajan P (2000) Studies on the biology of the scads, Decapterus russelli and Decapterus macrosoma at Vizhinjam, southwest coast of India. Indian J Fish 47:291-300

Batta-Lona PG, Galindo-Sánchez CE, Arteaga MC, Robles-Flores J, Jiménez-Rosenberg SPA (2019) DNA barcoding and morphological taxonomy: identification of lanternfish (Myctophidae) larvae in the Gulf of Mexico. Mitochondrial DNA Part A 30:2,375-2,383. https://doi.org/10.1080/24701394.2018.1538364

Beckley LE, Holliday D, Sutton AL, Weller E, Olivar MP, Thompson PA (2019) Structuring of larval fish assemblages along a coastaloceanic gradient in the macro-tidal, tropical Eastern Indian Ocean. Deep Sea Res. II Top Stud Oceanogr 161:105-119. https://doi.org/ 10.1016/j.dsr2.2018.03.008

Beldade R, Borges R, Gonçalves EJ (2006) Depth distribution of nearshore temperate fish larval assemblages near rocky substrates. J Plankton Res 28:1003-1013. https://doi.org/10.1093/plankt/fbl035

Borges R, Beldade R, Gonçalves EJ (2007) Vertical structure of very nearshore larval fish assemblages in a temperate rocky coast. Mar Biol 151:1349-1363. https://doi.org/10.1007/s00227-006-0574-z

Burrows M, Browning JS, Breitbart M, Murawski SA, Peebles EB (2019) DNA barcoding reveals clear delineation between spawning sites for neritic versus oceanic fishes in the Gulf of Mexico. Fish Oceanogr 28(2):228-239. https://doi.org/10.1111/fog.12404 
Carpenter KE (1988) Fusiliers of the world An annotated and illustrated catalogue of Caesionid species known to date FAO species catalogue Vol 8. $76 \mathrm{pp}$

Cohen DM (1990) Bathylagidae. In: Quéro JC, Hureau JC, Karrer C, Post A, Saldanha L (eds) Check-list of the fishes of the eastern tropical Atlantic (CLOFETA), vol 1. JNICT, Lisbon; SEI, Paris; and UNESCO, Paris, pp 239-240

Catul V, Gauns M, Karuppasamy PK (2011) A review on mesopelagic fishes belonging to the family Myctophidae. Rev Fish Biol Fisheries 21(3):339-354. https://doi.org/10.1007/s11169-010-9176-4

Collet A, Durand JD, Desmarais E, Cerqueir F, Cantinelli T, Valade P, Ponton D (2017) DNA barcoding post-larvae can improve the knowledge about fish biodiversity: an example from La Reunion, SW Indian Ocean. Mitochondrial DNA Part A. https://doi.org/10. 1080/24701394.2017.1383406

Dalpadado P, Gjøsæter J (1993) Lanternfishes (Myctophidae) in Sri Lankan waters. Asian Fish Sci 9:161-168

de Bruin GH, Russell BC, Bogusch A (1994) The marine fishery resources of Sri Lanka. Food and Agriculture Organization (FAO), Rome

de Vos A, Pattiaratchi CB, Wijeratne EMS (2014) Surface circulation and upwelling patterns around Sri Lanka. Biogeosciences 11:5909-5930. https://doi.org/10.5194/bg-11-5909-2014

Di Dario F (2018) Sardinella sindensis. IUCN red list of threatened species 2018:e.T46075339A46664244

FAO (2001) Report of the trilateral workshop on lanternfish in the Gulf of Oman. FAO Fish Rep No. 665, Rome. http://www.fao.org/3/ y2967e/y2967e.pdf

Fox CJ, Taylor M, Pereyra RT, Villasana MI, Rico C (2005) TaqMan DNA technology confirms likely overestimation of cod (Gadus morhua L.) egg abundance in the Irish Sea: implications for the assessment of the cod stock and mapping of spawning areas using egg-based methods. Mol Ecol 14:879-884. https://doi.org/10.1111/ j.1365-294x.2005.02439.x

Fricke R (2002) Annotated checklist of the dragonet families Callionymidae and Draconettidae (Teleostei: Callionymoidei), with comments on Callionymid fish classification. Stuttgarter Beitr NaturK Ser A (Biol) 645:1-103

Froese R, Pauly D (2019) FishBase. World Wide Web electronic publication https://www.fishbase.org version (12/2019)

Ganga U, Jinesh PT, Beni N (2015) A note on the ribbonfish Trichiurus auriga. Mar Fish Infor Serv T \& E ser 226:20-21

Gloerfelt-Tarp T, Kailola PJ (1984) Trawled fishes of southern Indonesia and northwestern Australia. Australian Development Assistance Bureau, Sydney, Australia, pp 407

Harden-Jones FR (1968) Fish migration. Edward Arnold, London

Herath D, Perera H, Hettiarachchi G (2019) Some biological aspects and molecular variations in frigate tuna, Auxis thazard of the coastal waters around Sri Lanka. J Natl Sci Found Sri Lanka 47(3):333340. https://doi.org/10.4038/jnsfsr.v47i3.9427

Hildago M, Browman H (2019) Developing the knowledge base needed to sustainably manage mesopelagic resources. ICES J Mar Sci 76: 609-615. https://doi.org/10.1093/icesjms/fsz067

Hoese DF, Larson HK (1994) Revision of the Indo-Pacific gobiid fish genus Valenciennea, with descriptions of seven new species. IndoPacific Fishes 23, Honolulu Hawaii, Bishop Museum

Holleman W, Connell AD, Carpenter KE (2013) Caesio xanthalytos, a new species of fusilier (Perciformes: Caesionidae) from the western Indian Ocean, with records of range extensions for several species of Caesionidae. Zootaxa 3702:262-272. https://doi.org/10.11646/ zootaxa.3702.3.4

Hood RR, Beckley LE, Wiggert JD (2017) Biogeochemical and ecological impacts of boundary currents in the Indian Ocean. Progr Oceanogr 156:290-325. https://doi.org/10.1016/j.pocean.2017.04. 011
Hubert N, Espiau B, Meyer C, Planes S (2015) Identifying the ichthyoplankton of a coral reef using DNA barcodes. Mol Ecol Resour 15:57-67. https://doi.org/10.1111/1755-0998.12293

Hutchings L, Beckley LE, Griffiths MH, Roberts MJ, Sundby S, van der Lingen C (2002) Spawning on the edge: spawning grounds and nursery areas around the southern African coastline. Mar Freshw Res 53:307-318. https://doi.org/10.1071/MF01147

ICES (2019) The working group on the integrated assessments of the Barents Sea (WGIBAR). ICES Sci Rep 1:42. 157 pp. https://doi. org/10.17895/ices.pub.5536

Isari S, Pearman JK, Casas L, Michell CT, Curdia J, Berumen ML, Irigoien X (2017) Exploring the larval fish community of the central Red Sea with an integrated morphological and molecular approach. PLoS One 12(8):e0182503. https://doi.org/10.1371/journal.pone. 0182503

Joshi KK, Sreeram MP, Zacharia PU, Abdussamad EM, Varghese M, Mohammed Habeeb OMMJ et al (2016) Check list of fishes of the Gulf of Mannar ecosystem, Tamil Nadu, India. J Mar Biol Assoc India 58(1):34-54. https://doi.org/10.6024/jmbai.2016.58.1.1895-05

Karunasinghe WPN, Fernando PAT, Samaraweera EKV (2000) Status of the gill net fishery for the small pelagic fish species in western, south-western and southern coastal waters of Sri Lanka during 1995-1997. Journal of the National Aquatic Resources Research and Development Agency of Sri Lanka 36:66-79. http:// aquaticcommons.org/id/eprint/17320

Krakstad JO, Jayasinghe P, Totland A, Dalpadado P, Søiland H, Cervantes D et al (2018) Survey of regional resources and ecosystems of the Bay of Bengal: part 1 Sri Lanka, 24 June- 16 July 2018. NORAD-FAO programme GCP/GLO/690/NOR, Cruise reports Dr. FRIDTJOF NANSEN, EAF-Nansen/CR/2018/8 162 pp

Kumara TPB, Dalpathadu KR (2012) Provisional checklist of marine fish of Sri Lanka. In: Weerakoon DK, Wijesundara S (eds) The National Red List 2012 of Sri Lanka; conservation status of the Fauna and Flora. Sri Lanka, Ministry of Environment, Colombo, pp 401-430

Lakra WS, Verma MS, Goswami M, Lal KK, Mohindra V, Punia P, Gopalakrishnan A, Singh KV, Ward RD, Hebert P (2011) DNA barcoding Indian marine fishes. Mol Ecol Resour 11:60-71. https://doi.org/10.1111/j.1755-0998.2010.02894.x

Larson H (2016a) Valenciennea puellaris. The IUCN Red List of Threatened Species 2016: e.T193068A2190891. https://doi.org/10. 2305/IUCN.UK.2016-3.RLTS.T193068A2190891.en. Downloaded on 20 October 2019

Larson H (2016b). Valenciennea wardii. The IUCN Red List of Threatened Species 2016: e.T68333312A72474262.https://doi.org/ 10.2305/IUCN.UK.20163.RLTS.T68333312A72474262.en. Downloaded on 19 October 2019

Legendre P, Legendre LF (2012) Numerical ecology (Vol. 24). Elsevier, Amsterdam

Leis JM (2015) Taxonomy and systematics of larval Indo-Pacific fishes: a review of progress since 1981. Ichthyol Res 62:9-28. https://doi. org/10.1007/s10228-014-0426-7

Leis JM, Carson-Ewart BM (2004) The larvae of Indo-Pacific coastal fishes: a guide to identification (Fauna Malesiana handbook 2), 2nd edn. Brill, Leiden

Lirdwitayaprasit P, Nuangsang C, Puewkhao P, Rahman MJ, Oo UAH, Sien UAW (2008) Composition, abundance, and distribution of fish larvae in the Bay of Bengal. SEAFDEC, Thailand:93-124

Long BG, Amarasiri C, Rajasurya A, Dissanayaka DCT, Liyanage KUSP, Jayasinghe RPPK, Athukoorala AASH, Karunathilaka KMB, Fernando HSG, Fernando TD (2010) Near shore fisheries status atlas, north west, south and east coast aquarium fish, chank, lobster, shrimp and sea cucumber fisheries of Sri Lanka, volume 1. National Aquatic Resource Research and Development Agency, Crow Island, Mattakkuliya, Colombo 15, Sri Lanka. GCP/SRL/ 054/CAN, pp 1-234 
Martin RP, Olson EE, Girard MG, Smith WL, Davis MP (2018) Light in the darkness: new perspective on lanternfish relationships and classification using genomic and morphological data. Mol Phylogenet Evol 121:71-85. https://doi.org/10.1016/j.ympev.2017.12.029

Mateos-Rivera A, Skern-Mauritzen R, Dahle G, Sundby S, Mozfar B, Thorsen A, Wehde H, Krafft BA (2020) Comparison of visual and molecular taxonomic methods to identify ichthyoplankton in the North Sea. Limnol Oceanogr-Meth 18:599-605. https://doi.org/10. 1002/lom3.10387

Mishra SS, Chakraborty P, Saren SC, Sengupta A (2018) First record of Lagocephalus guentheri Miranda Riberio 1915 (Tetraodontiformes: Tetraodontidae) from the West Coast of India. Rec Zool Surv India 118:91-96. https://doi.org/10.26515/rzsi/v118/i1/2018/122386

Montero-Pau J, Gómez A, Joaquín Muñoz J (2008) Application of an inexpensive and high-throughput genomic DNA extraction method for the molecular ecology of zooplanktonic diapausing eggs. Limnol Oceanogr Methods 6:218-222. https://doi.org/10.4319/lom.2008.6. 218

Motomura H, Matsuura K, Khan M (2018) Synanceia verrucosa. The IUCN red list of threatened species 2018:e.T114180058A116389089. https:// doi.org/10.2305/IUCN.UK.2018-2.RLTS.T114180058A116389039.en

Munroe T, Costa M, Kobyliansky S, Arnold R (2015) Ceratias holboelli. The IUCN Red List of Threatened Species 2015: e.T18127741A21910125.https://doi.org/10.2305/IUCN.UK.20154.RLTS.T18127741A21910125.en. (accessed 01 November 2019)

Nair RJ (2008) Taxonomic account of Marcia's anthias, Pseudanthias marcia Randall \& Hoover, 1993 (Serranidae: Anthiinae), a new record from India. J Mar Biol Ass India 50:98-102

Nair RJ, Gopalakrishnan A (2015) A taxonomic note on Crossorhombus azureus (Alcock 1889) (family: Bothidae, order: Pleuronectiformes) from the south-west coast of India. Indian J Fish 62:87-91

Nair RJ, Kumar SD (2018) Overview of the fish diversity of Indian waters. In: Vijayagopal P, Peter R (eds) Training manual in the framework of the project: DBT sponsored three months national training in molecular biology and biotechnology for fisheries professionals. Central Marine Fisheries Research Institute, Kochi, pp $35-66$

Olivar MP, Contreras T, Hulley PA, Emelianov M, López-Pérez C, Tuset V, Castellón A (2018) Variation in the diel vertical distributions of larvae and transforming stages of oceanic fishes across the tropical and equatorial Atlantic. Prog Oceanogr 160:83-100. https://doi.org/ 10.1016/j.pocean.2017.12.005

Pezold FL, Larson HK (2015) A revision of the fish genus Oxyurichthys (Gobioidei: Gobiidae) with description of four new species. Zootaxa 3988(1):1. https://doi.org/10.11646/zootaxa.3988.1.1

Pielou EC (1975) Ecological diversity. Wiley, New York

Pillai NGK, Satheeshkumar P (2012) Biology, fishery, conservation and management of Indian Ocean tuna fisheries. Ocean Sci J 47:411-433. https://doi.org/10.1007/s12601-012-0038-y

Pillai PP, Silas EG (1979) Distribution and biology of the skipjack tuna Katsuwonus pelamis taken by the longline fishery in the Indian ocean. Mar Biol Ass India 21:147-170

Potier M, Romanov E, Cherel Y, Sabatié R, Zamorov V, Ménard F (2008) Spatial distribution of Cubiceps pauciradiatus (Perciformes: Nomeidae) in the tropical Indian Ocean and its importance in the diet of large pelagic fishes. Aquat Living Resour 21: 123-134. https://doi.org/10.1051/alr:2008026

R Core Team (2018) R: a language and environment for statistical computing. R Foundation for Statistical Computing, Vienna, Austria URL https://www.R-project.org/

Rajasuriya A (2013) Field guide to reef fishes of Sri Lanka. IUCN Sri Lanka Office 1, Colombo, pp 1-104

Rajasuriya A (2014) Field guide to reef fishes of Sri Lanka. Vol. 2 Colombo: IUCN Sri Lanka Office. 1-101

Rathnasuriya MIG, Mateos-Rivera A, Bandara AGGC, Skern-Mauritzen R, Jayasinghe RPPK, Krakstad JO, Dalpadado P (2019) DNA barcoding confirms the first record of a Desmodema polystictum (Ogilby, 1898) egg and all-time high adult catches in the Indian Ocean. Mar Biodivers Rec 12:22. https://doi.org/10.1186/s41200019-0180-7

Reglero P, Tittensor DP, Álvarez-Berastegui D, Aparicio-González A, Worm B (2014) Worldwide distributions of tuna larvae: revisiting hypotheses on environmental requirements for spawning habitats. Mar Ecol Prog Ser 501:207-224. https://doi.org/10.3354/ meps 10666

Rodriguez JM, Alemany F, Garcia A (2017) A guide to the eggs and larvae of 100 common western Mediterranean Sea bony fish species. FAO, Rome

Roos D, Roux O, Conand F (2007) Notes on the biology of the bigeye scad, Selar crumenophthalmus (Carangidae) around Reunion Island, Southwest Indian Ocean. Sci Mar 71:137-144. https://doi. org/10.3989/scimar.2007.71n1137

Sakaue J, Akino H, Endo M, Ida H, Asahida T (2016) Temporal and spatial site sharing during spawning in snappers Symphorichthys spilurus and Lutjanus bohar (Pisces: Perciformes: Lutjanidae) in waters around Peleliu island, Palau. Zool Stud 55:1-15. https://doi. org/10.6620/ZS.2016.55-44

Sassa C, Moser HG, Kawaguchi K (2002) Horizontal and vertical distribution patterns of larval myctophid fishes in the Kuroshio current region. Fish Oceanogr 11:1-10. https://doi.org/10.1046/j.13652419.2002.00182.x

Sebastine M, Bineesh KK, Abdussamad EM, Pillai NGK (2013) Myctophid fishery along the Kerala coast with emphasis on population characteristics and biology of the headlight fish, Diaphus watasei Jordan \& Starks, 1904. Indian J Fish 60:7-11

Shannon CE, Weaver W (1949) The mathematical theory of communication. University of Illinois Press, Urbana-Champaign

Song Q, Gordon AL, Visbeck M (2004) Spreading of the Indonesian Throughflow in the Indian Ocean. J Phys Oceanogr 34:772-792. https://doi.org/10.1175/1520-0485(2004)034<0772:SOTITI >2.0. $\mathrm{CO} ; 2$

Steinke D, Connell AD, Hebert DN (2016) Linking adults and immatures of South African marine fishes. Genome 59:959-967. https://doi. org/10.1139/gen-2015-0212

Stequert B, Marsac F (1989) Tropical tuna - surface fisheries in the Indian Ocean. FAO Fish Tech Pap No 282 pp 238 FAO, Rome,

Subarna D (2018) The effect of monsoon variability on fish landing in the Sadeng Fishing Port of Yogyakarta. Indonesia IOP Conf Ser Earth Environ Sci 139 012027. https://doi.org/10.1088/1755-1315/139/1/ 012027

Tomczak M, Godfrey JS (1994) The Indian Ocean. In: Regional oceanography: an introduction. Pergamon, Oxford

Uiblein F, Heemstra PC (2010) A taxonomic review of the Western Indian Ocean goatfishes of the genus Upeneus (family Mullidae), with descriptions of four new species. Smithiana Bull 11:35-71. http://hdl.handle.net/11250/117221

Uiblein F, Heemstra PC (2011) Description of a new goatfish species, Upeneus randalli $\mathrm{n}$. sp. (Mullidae), from the Persian Gulf, with remarks and identification keys for the genus Upeneus. Sci Mar 3:585-594. https://doi.org/10.3989/scimar.2011.75n3585

Valinassab T, Pierce GJ, Johannesson K (2007) Lantern fish (Benthosema pterotum) resources as a target for commercial exploitation in the Oman Sea. J Appl Ichthyol 23:573-577. https://doi.org/ 10.1111/j.1439-0426.2007.01034.x

Velamala GR, Naranji KM, Babu RK (2017) New record of blue striped snapper, Lutjanus Notatus (Cuvier, 1828) (family: Lutjanidae), from Indian waters. J Aquac Mar Biol 5:00113. https://doi.org/10.15406/ jamb.2017.05.00113

Victor BC (2016) Two new species in the spike-fin fairy-wrasse species complex (Teleostei: Labridae: Cirrhilabrus) from the Indian Ocean. J Ocean Sci Found 23:21-50. https://doi.org/10.5281/zenodo.163217 
Wlliams JT (2014) Trichiurus auriga. The IUCN red list of threatened species 2014:e.T46079924A48376643. https://doi.org/10.2305/ IUCN.UK.2014-3.RLTS.T46079924A48376643.en

Yapa KKAS (2000) Seasonal variability of sea surface chlorophyll-a of waters around Sri Lanka. J Earth Syst Sci 109:427-432. https://doi. org/10.1007/BF02708330
Zemlak TS, Ward RD, Connell AD, Holmes BH, Hebert PDN (2009) DNA barcoding reveals overlooked marine fishes. Mol Ecol Resour 9:237-224. https://doi.org/10.1111/j.1755-0998.2009.02649.x

Publisher's note Springer Nature remains neutral with regard to jurisdictional claims in published maps and institutional affiliations. 